\title{
CORREÇÃO DA ACIDEZ DO SOLO E APORTE DE METAIS PESADOS PELA APLICAÇÃO DE ESCÓRIA BÁSICA DE ACIARIA
}

\author{
SOIL CHEMICAL PROPERTIES CHANGES BY APPLICATION OF STEEL BASIC \\ $S L A G$
}

\author{
Márcio do Sacramento WALLY ${ }^{\mathbf{1}}$ Carlos Alberto BISSANI ${ }^{\mathbf{1}}$; \\ Viviane Pereira dos SANTOS ${ }^{1}$; Leandro BORTOLON ${ }^{2}$, \\ Elisandra Solange Oliveira BORTOLON ${ }^{2}$; Robson ANDREAZZA ${ }^{3}$; Clesio GIANELLO $^{1}$ \\ 1. Departamento de Solos, Faculdade de Agronomia; Universidade Federal do Rio Grande do Sul; Porto Alegre, RS, Brasil; 2. Embrapa \\ Pesca e Aquicultura; EMBRAPA; TO, Brazil; 3. Centro de Engenharias; Universidade Federal de Pelotas; Pelotas, RS, Brasil. \\ robsonandreazza@yahoo.com.br;
}

\begin{abstract}
RESUMO: A fabricação de aço está expandindo sua produção, o que vem aumentando a quantidade de resíduos sólidos gerados. As escórias básicas de aciaria apresentam características de corretivo da acidez do solo e fonte de nutrientes para as plantas, porém apresentam alguns metais pesados em sua constituição. O presente trabalho teve como objetivo avaliar o efeito da aplicação de escória de siderurgia na correção da acidez do solo e no aporte de metais pesados, como manganês $(\mathrm{Mn})$, cobre $(\mathrm{Cu})$, zinzo $(\mathrm{Zn})$, cádmio $(\mathrm{Cd})$, cromo $(\mathrm{Cr})$ e níquel $(\mathrm{Ni})$. O estudo foi conduzido em amostras de um Argissolo Vermelho Distrófico típico, dispostas em colunas e mantidas a céu aberto, em delineamento experimental de blocos casualizados, com quatro repetições. Os tratamentos consistiram em doses diferentes de escória de siderurgia com diferentes granulometrias, incluindo tratamentos com solo sob condição natural (controle) e corrigido (carbonato de cálcio e magnésio e adubação com nitrogênio, fósforo e potássio). Aos 12, 18 e 24 meses após a aplicação dos tratamentos foram coletadas amostras de solo das colunas para avaliação da reatividade da escória. A escória básica de aciaria finamente moída teve efeito similar ao carbonato na correção do pH do solo, no aumento da CTC efetiva e da saturação de bases. Partículas com diâmetro até $15 \mathrm{~mm}$ também foram efetivas para aumentar os valores desses atributos. As maiores doses de escória aumentaram os teores de $\mathrm{Mn}, \mathrm{Cr}$ e Ni no solo em relação ao controle. Os resultados obtidos neste estudo, demonstram que a escória de siderurgia tem potencial para a utilização na correção do pH do solo.
\end{abstract}

PALAVRAS-CHAVE: Acidez do solo. Resíduo industrial. Nutrientes.

\section{INTRODUÇÃO}

Nos últimos anos, tem aumentado a demanda de produtos derivados do ferro e do aço, impulsionando o crescimento do setor siderúrgico nacional. Assim, como a maioria dos processos industriais, a siderurgia é geradora de grande quantidade de resíduos. Entre os resíduos da fabricação do aço estão às escórias de siderurgia. As opções de reutilização destes resíduos são muito restritas, o que ocasiona seu acúmulo nas indústrias.

A escória é um produto não-metálico, composto essencialmente de silicatos e aluminossilicatos de cálcio e outras bases (GUIMARÃES, 1967). Sua composição química é formada por óxidos de cálcio, magnésio, silício, ferro e manganês, resultante da constituição química da matéria-prima e do material refratário utilizado nas paredes do forno. Devido suas características físicas, a escória de aciaria tem sido utilizada em diferentes composições na construção civil (PINTO JUNIOR et al., 2011; ARRIVABENE et al., 2012; ROSSA; PORTELLA, 2012), bem como outras utilizações como em misturas de base asfáltica, contudo, há uma preocupação com o impacto dos metais pesados no ambiente (GARCIA-GUINEA et al., 2010).

O caráter alcalino das escórias possibilita sua utilização como corretivo da acidez do solo e também como fonte de alguns nutrientes para as plantas (PEREGRINA et al., 2008). Porém, ainda há uma necessidade maior de pesquisar mais a utilização desse resíduo na agricultura, e seus efeitos para a mesma. A maior restrição para a utilização destes produtos seria a presença de metais pesados em sua constituição (GARCIA-GUINEA et al., 2010).

Considerando que os solos tropicais e subtropicais são ácidos e de baixa fertilidade natural, a utilização de resíduos com características corretivas ou fertilizantes é uma alternativa de disposição final (PEREGRINA et al., 2008; NEGIM et al., 2010). Ao exemplo dos calcários agrícolas, a eficiência da escória em reagir no solo é inversamente relacionada ao tamanho das partículas. CAMARGO (1972), usando uma escória na dose de duas vezes o teor de $\mathrm{Al}^{3+}$, encontrou neutralização deste elemento tanto mais rápida quanto menor o tamanho de partículas do material. FORTES (1993) estudou duas escórias de siderurgia em diferentes frações granulométricas, variando de $0,84 \mathrm{~mm}$ até 
menor que $0,05 \mathrm{~mm}$ e concluiu que os melhores resultados ocorreram com as partículas menores que 0,15 mm, aos 90 dias de incubação. Nota-se, pelos resultados da literatura, a importância da moagem fina da escória no aumento da superfície específica do material para favorecer sua reação no solo.

A escória pode trazer alguns benefícios extras a fertilidade do solo, não apenas pelo aumento do $\mathrm{pH}$ e cátions trocáveis, mas por conter em sua constituição química alguns micronutrientes como Mn, Cu, Zn e Fe (BARBER, 1967). Prado et al. (2001), aplicando quatro doses crescentes de escória em pré-plantio de cana-de-açúcar, observaram que a aplicação da escória de siderurgia promoveu um efeito residual favorável na disponibilidade de $\mathrm{Fe}, \mathrm{Cu}$ e $\mathrm{Zn}$ disponível no solo. Deste modo, a aplicação da escória de siderurgia pode corrigir a acidez do solo e, ao mesmo tempo, fornecer nutrientes. Sendo assim, haveria vantagem em relação ao calcário, uma vez que este não apresenta em sua composição a quantidade de micronutrientes que há na escória (VALADARES et al., 1974).

Este trabalho se fundamenta nas hipóteses de que a escória básica de aciaria atua como corretivo de acidez do solo, proporcionando aumento na concentração de nutrientes e metais pesados, sendo que a eficiência depende da dose e do tamanho de partículas do material aplicado. Sendo assim, o objetivo do trabalho foi avaliar a eficiência da correção da acidez de um argissolo e a liberação de nutrientes e de metais pesados pela aplicação de escória básica de aciaria. Além disso, foram testadas diferentes doses e tamanhos de partículas ao longo do tempo.

\section{MATERIAL E MÉTODOS}

Este trabalho teve início em maio de 2003 e foi desenvolvido nas dependências do Departamento de Solos da Faculdade de Agronomia, UFRGS, dando-se sequência a um experimento instalado em abril de 2002. O experimento foi conduzido em microparcelas a céu aberto, constituídas de colunas de PVC com $20 \mathrm{~cm}$ de diâmetro e $35 \mathrm{~cm}$ de altura, com capacidade para $10 \mathrm{dm}^{3}$ de solo, fechada na extremidade inferior com disco de madeira, impermeabilizada com resina epoxi e com sistema de escoamento do excesso de água. O solo utilizado foi um Argissolo Vermelho distrófico típico - PVd, na quantidade de $8 \mathrm{~kg}$ por unidade experimental, proveniente da unidade de mapeamento São Jerônimo (STRECK et al. 2008), coletado em área de campo nativo, na camada de $0-20 \mathrm{~cm}$ de profundidade, na Estação Experimental Agronômica da UFRGS, no município de Eldorado do Sul, RS. Este foi seco ao ar e tamisado em peneira de $4 \mathrm{~mm}$ de diâmetro de orifícios. As características químicas deste solo encontram-se na Tabela 1.

Tabela 1. Caracterização físico-química original do solo utilizado no experimento

\begin{tabular}{|c|c|c|c|}
\hline Determinação $^{(1)}$ & Valor & Determinação $^{(1)}$ & Valor \\
\hline Argila, $\mathrm{g} \mathrm{kg}^{-1}$ & 300 & CTC efetiva, $\mathrm{cmol}_{\mathrm{c}} \mathrm{dm}^{-3}$ & 3,2 \\
\hline Matéria orgânica, $\mathrm{g} \mathrm{dm}^{-3}$ & 22 & CTC pH 7,0, $\mathrm{cmol}_{\mathrm{c}} \mathrm{dm}^{-3}$ & 5,9 \\
\hline $\mathrm{pH}\left(\mathrm{H}_{2} \mathrm{O}\right)$ & 4,8 & Saturação por bases, \% & 44 \\
\hline pH SMP (Índice SMP) & 6,1 & Saturação por alumínio, \% & 19 \\
\hline $\mathrm{P}\left(\right.$ Mehlich -1), $\mathrm{mg} \mathrm{dm}^{-3}$ & 10 & Nec. calcário (pH 6,5), $\mathrm{t} \mathrm{ha}^{-1}$ & 4,3 \\
\hline $\mathrm{K}$ (Mehlich-1), $\mathrm{mg} \mathrm{dm}^{-3}$ & 162 & $\mathrm{~S}\left(\mathrm{~S}^{-\mathrm{SO}_{4}}{ }^{2-}\right), \mathrm{mg} \mathrm{dm}^{-3}$ & 8,1 \\
\hline $\mathrm{Al}$ trocável, $\mathrm{cmol}_{\mathrm{c}} \mathrm{dm}^{-3}$ & 0,6 & $\mathrm{Cu}\left(\mathrm{HCl} 0,1 \mathrm{~mol} \mathrm{~L}^{-1}\right), \mathrm{mg} \mathrm{dm}^{-3}$ & 0,9 \\
\hline Ca trocável, $\mathrm{cmol}_{\mathrm{c}} \mathrm{dm}^{-3}$ & 1,4 & $\mathrm{Zn}\left(\mathrm{HCl} 0,1 \mathrm{~mol} \mathrm{~L}^{-1}\right), \mathrm{mg} \mathrm{dm}^{-3}$ & 1,4 \\
\hline $\mathrm{Mg}$ trocável, $\mathrm{cmol}_{\mathrm{c}} \mathrm{dm}^{-3}$ & 0,8 & $\mathrm{~B}$ (água quente), $\mathrm{mg} \mathrm{dm}^{-3}$ & 0,8 \\
\hline $\mathrm{H}+\mathrm{Al}, \mathrm{cmol}_{\mathrm{c}} \mathrm{dm}^{-3}$ & 3,3 & $\mathrm{Mn}\left(\mathrm{KCl} 1 \mathrm{~mol} \mathrm{~L}^{-1}\right), \mathrm{mg} \mathrm{dm}^{-3}$ & 8,0 \\
\hline
\end{tabular}


O resíduo utilizado nos tratamentos foi à escória básica de aciaria, proveniente do processo de

fabricação de aço inoxidável de uma siderúrgica estabelecida no Rio Grande do Sul. A coleta foi realizada por amostragem segundo as normas da NBR 10.007 (amostragem de resíduos), na área de estocagem da escória, totalizando $10 \mathrm{~kg}$. Parte da amostra foi moída e tamisada, de forma a apresentar as granulometrias de $0,149 \mathrm{~mm}, 0,5 \mathrm{~mm}$, $2,0 \mathrm{~mm}$ e $15,0 \mathrm{~mm}$. A escória foi analisada quimicamente quanto aos teores totais dos principais elementos, a partir de digestão de amostra finamente moída, pelo método EPA 3052 (USEPA, 1996b), sendo as concentrações totais dos elementos Cálcio, Magnésio, Ferro, Manganês, Cobre, Zinco, Cádmio, Cromo, Níquel e Chumbo determinadas por espectroscopia de absorção atômica; Fósforo, por espectroscopia de absorção molecular; e Potássio e Sódio, por fotometria de chama. A determinação do poder de neutralização $(\mathrm{PN})$ foi feita por ataque ácido, segundo TEDESCO et al. (1995). A caracterização da escória é apresentada na Tabela 2.

Tabela 2. Características químicas do resíduo utilizado no experimento.

\begin{tabular}{lclc}
\hline Determinação & Valor $^{(\mathbf{1})}$ & Determinação( & Valor \\
\hline Fósforo, $\mathrm{P}_{2} \mathrm{O}_{5}, \mathrm{mg} \mathrm{kg}^{-1}$ & $<0,37$ & Cádmio, $\mathrm{mg} \mathrm{kg}^{-1}$ & 2,3 \\
Potássio, $\mathrm{K}_{2} \mathrm{O}, \mathrm{mg} \mathrm{kg}^{-1}$ & 132,5 & Chumbo, mg kg & 6,2 \\
Cálcio, $\mathrm{CaO}, \mathrm{g} \mathrm{kg}^{-1}$ & 320 & Cobre, $\mathrm{mg} \mathrm{kg}^{-1}$ & 21,4 \\
Magnésio, $\mathrm{MgO}, \mathrm{g} \mathrm{kg}^{-1}$ & 79 & Cromo, $\mathrm{g} \mathrm{kg}^{-1}$ & 4,62 \\
Sódio, $\mathrm{mg} \mathrm{kg}^{-1}$ & 120,7 & Manganês, $\mathrm{g} \mathrm{kg}^{-1}$ & 3,41 \\
Poder de neutralização (PN), \% & 70 & Níquel, mg kg & 93,8 \\
Ferro, $\mathrm{g} \mathrm{kg}^{-1}$ & 12,1 & Zinco, $\mathrm{mg} \mathrm{kg}^{-1}$ & 56,4 \\
\hline
\end{tabular}

${ }^{(1)}$ Os valores expressam as concentrações totais.

Os tratamentos estudados foram:

T1) solo na condição natural - Controle;

T2) Com adição de calário para atingir pH

$6,5 \quad\left(\mathrm{CaCO}_{3}+\mathrm{MgCO}_{3} ; \quad\right.$ PRNT $\left.100 \%\right) \quad-$ $\mathrm{CaCO}_{3}+\mathrm{MgCO}_{3}$;

T3) Com adição de calário para atingir pH $6,5\left(\mathrm{CaCO}_{3}+\mathrm{MgCO}_{3}\right.$; PRNT $\left.100 \%\right)+$ adubação $\mathrm{NPK}-\mathrm{CaCO}_{3}+\mathrm{MgCO}_{3}+\mathrm{NPK}$;

T4) Escória (1), com diâmetro de partículas $<0,149 \mathrm{~mm}$, na quantidade de 1x SMP para atingir pH 6,5 - Escória nível 1

T5) Escória (1), com diâmetro de partículas $<0,149 \mathrm{~mm}$, na quantidade de 1x SMP para atingir pH 6,5 + NPK - Escória nível 1+ NPK

T6) Escória (2) com 2/3 das partículas de diâmetro entre $0,149 \mathrm{~mm}$ e $0,5 \mathrm{~mm}$ e $1 / 3<0,149$ $\mathrm{mm}$, na quantidade de 2x SMP para atingir $\mathrm{pH}$ 6,5 + NPK - Escória nível 2 + NPK;

T7) Escória (1), com diâmetro de partículas $<0,149 \mathrm{~mm}$, na quantidade de 1x SMP para atingir pH 6,5 + Nitrogênio - Escória nível $1+\mathrm{N}$

T8) Escória (5), com diâmetro de partículas entre $0,5 \mathrm{~mm}$ e $2,0 \mathrm{~mm}$, na quantidade de $5 \mathrm{x}$ SMP para atingir pH 6,5 + calcário para atingir $\mathrm{pH} 6,5$ + NPK - Escória nível 5 + T3;

T9) Escória (10), com diâmetro de partículas entre $2,0 \mathrm{~mm}$ e $15,0 \mathrm{~mm}$ na quantidade de 10x SMP para atingir $\mathrm{pH} 6,5$ + calcário para atingir pH 6,5 + NPK - Escória nível 10 + T3.
No tratamento com calcário, usou-se uma misura de $\mathrm{CaCO}_{3}+\mathrm{MgCO}_{3}$ (3:1 em peso), com PRNT 100\%, para obter o valor de $\mathrm{pH} 6,5$, pela metodologia do índice SMP, conforme a análise de solo e as recomendações de calagem para estados do RS e SC (SBCS/NRS, 1995). Pelo mesmo critério de valor de $\mathrm{pH}$ a atingir e considerando um valor de neutralização do resíduo de $60 \%$, obtido em experimento de incubação (LUZ et al., 2002), foram estabelecidas as doses de escória $(1,2,5 \mathrm{ou}$ 10 vezes a necessidade de corretivo para atingir $\mathrm{pH}$ 6,5, conforme os tratamentos) (Tabela 3). A adição do Fósforo, Potássio e Nitrogênio foram feitos mediante as aplicações de superfosfato triplo, cloreto de potássio e uréia, respectivamente. As quantidades adicionadas de corretivo, resíduo e fertilizante foram calculadas utilizando-se como base de cálculo a massa de $2,4 \times 10^{6} \mathrm{~kg}$ de solo seco por hectare.

Os tratamentos foram aplicados ao solo no dia 22 de abril de 2002. À exceção da adubação de cobertura, todos os materiais foram quantificados e aplicados individualmente aos respectivos vasos, sendo misturados e homogeneizados com a massa de solo. A partir de então, os vasos foram mantidos em área telada, a céu aberto, sob condição de chuva natural. Durante os cultivos, quando necessário, foi feita irrigação complementar, conforme a demanda hídrica das culturas. 
Tabela 3. Quantidades de calcário e escória de siderurgia aplicados em cada tratamento.

\begin{tabular}{lccccc}
\hline \multirow{2}{*}{ Tratamento } & $\begin{array}{c}\text { Calcário } \\
\left(\mathrm{CaCO}_{3}+\mathrm{MgCO}_{3}\right)\end{array}$ & \multicolumn{4}{c}{ Escória (diâmetros em mm de orifícios de peneiras) } \\
\cline { 3 - 6 } & - & $<0,149$ & $0,149-0,5$ & $0,5-2,0$ & $2,0-15,0$ \\
\hline 1) Controle & - & - & - & - & - \\
2) $\mathrm{CaCO}_{3}+\mathrm{MgCO}_{3}$ & 4,3 & - & - & - & - \\
3) $\mathrm{CaCO}_{3}+\mathrm{MgCO}_{3}+\mathrm{NPK}$ & 4,3 & - & - & - & - \\
4) Escória nível 1 & - & 7,2 & - & - & - \\
5) Escória nível 1+ NPK & - & 7,2 & - & - & - \\
6) Escória nível 2 + NPK & - & 9,6 & 4,2 & - & - \\
7) Escória nível 1 + N & - & 7,2 & - & - & - \\
8) Escória nível 5 + T3 & 4,3 & - & - & 36,0 & - \\
9) Escória nível 10 + T3 & 4,3 & - & - & - & 72,0 \\
\hline
\end{tabular}

A partir da instalação do experimento, foram conduzidos três cultivos sucessivos com gramíneas, dois no inverno (aveia branca - Avena sativa e aveia preta - Avena strigosa) e um no verão (milho - Zea mays) (dados não apresentados).

Após cada cultivo, o solo de cada vaso foi amostrado na camada de $0-20 \mathrm{~cm}$, aproximadamente $0,25 \mathrm{~kg}$, com trado calador. As amostragens foram realizadas em períodos de 12, 18 e 24 meses após a aplicação dos tratamentos ao solo. Em cada coleta, as amostras foram homogeneizadas, secas em estufa a $45^{\circ} \mathrm{C}$, moídas em moinho de martelos e tamisadas em peneira de $2 \mathrm{~mm}$ de aberturas. Nas amostras secas, foram realizadas as seguintes determinações, conforme metodologias descritas por TEDESCO et al. (1995): pH em água (relação 1:1), índice SMP; $\mathrm{H}+\mathrm{Al}$ (acidez potencial, por correlação com o índice SMP); fósforo e potássio disponíveis (Mehlich-1); cálcio, magnésio, alumínio e manganês trocáveis (extrator $\mathrm{KCl} 1 \mathrm{~mol} \mathrm{~L}^{-1}$ ); zinco e cobre extraíveis ( $\left.\mathrm{HCl} 0,1 \mathrm{~mol} \mathrm{~L}^{-1}\right)$. Nas amostras correspondentes aos 12 e 24 meses, foram também determinados os teores de cobre $(\mathrm{Cu})$, zinco $(\mathrm{Zn})$, manganês $(\mathrm{Mn})$, cádmio $(\mathrm{Cd})$, cromo $(\mathrm{Cr})$ e níquel $(\mathrm{Ni})$, pela metodologia EPA 3050b (USEPA, 1996a). Nos extratos obtidos, as determinações de fósforo e potássio foram feitas por espectrometria de absorção molecular (colorimetria) e fotometria de chama, respectivamente; todos os demais elementos foram determinados por espectrometria de absorção atômica (chama acetileno-óxido nitroso para Cromo e ar-acetileno para os demais). Com os dados disponíveis, foram calculados os valores de CTC efetiva, CTC a pH 7,0 e saturação por bases, conforme Tedesco et al. (1995).

A análise estatística seguiu o delineamento de blocos casualizados, com quatro repetições. A análise de variância (teste $F$ ) e a comparação de médias de tratamentos pelo Teste de Tukey a 5\% de probabilidade foram feitas com a utilização do software Sanest (ZONTA et al., 1984). Para alguns dados de solo, foram comparadas as médias de época de amostragem dentro de cada tratamento, a partir de efeito de interação.

\section{RESULTADOS E DISCUSSÃO}

Os valores de $\mathrm{pH}$ nos tratamentos com a aplicação de calcário e escória, determinados nas diferentes etapas, foram maiores do que os determinados no tratamento controle (Figura 1), demonstrando a eficiência destes dois corretivos na correção do pH do solo. Em geral, os valores obtidos foram próximos ao $\mathrm{pH}$ 6,5 determinado. A aplicação de escória na quantidade equivalente a 1 SMP para $\mathrm{pH}$ 6,5, com granulometria fina (tratamentos 4, $5 \mathrm{e}$ 7), proporcionou efeito semelhante ao carbonato na correção do $\mathrm{pH}$. Resultados similares foram obtidos por Prado et al. (2001) para uma escória de siderurgia e um calcário, incorporados no solo em pré-plantio de cana-de-açúcar e por Prado; Korndorfer (2003) para a escória aplicada em um Latossolo Vermelho e cultivado com milho.

A composição granulométrica da escória tem efeito na reatividade. No tratamento 9 , a adição do dobro da dose de escória em relação ao tratamento 8 não resultou em aumento do $\mathrm{pH}$. Porém, comparando-se estes dois tratamentos com os demais, se observou o efeito adicional significativo da escória na elevação do pH. Em ambos, a aplicação de calcário teve o objetivo de elevar o $\mathrm{pH}$ a 6,5 , mas os valores encontrados, já ao final de 12 meses, foram significativamente superiores aos tratamentos com apenas carbonato (2 e 3$)$.

Em geral, no decorrer do período de avaliação, foi observada significativa diminuição do $\mathrm{pH}$ do solo, do mesmo modo que o tratamento calcário + NPK (Figura 1). Constatou-se o maior decréscimo no trat. 7, no qual a escória foi complementada apenas com $\mathrm{N}$ e as plantas tiveram 
restrição ao crescimento, como reportado posteriormente. A acidificação do solo é um processo natural favorecido pelo uso de fertilizantes nitrogenados, lixiviação de bases, mineralização da matéria orgânica, etc. (BISSANI et al., 2004).

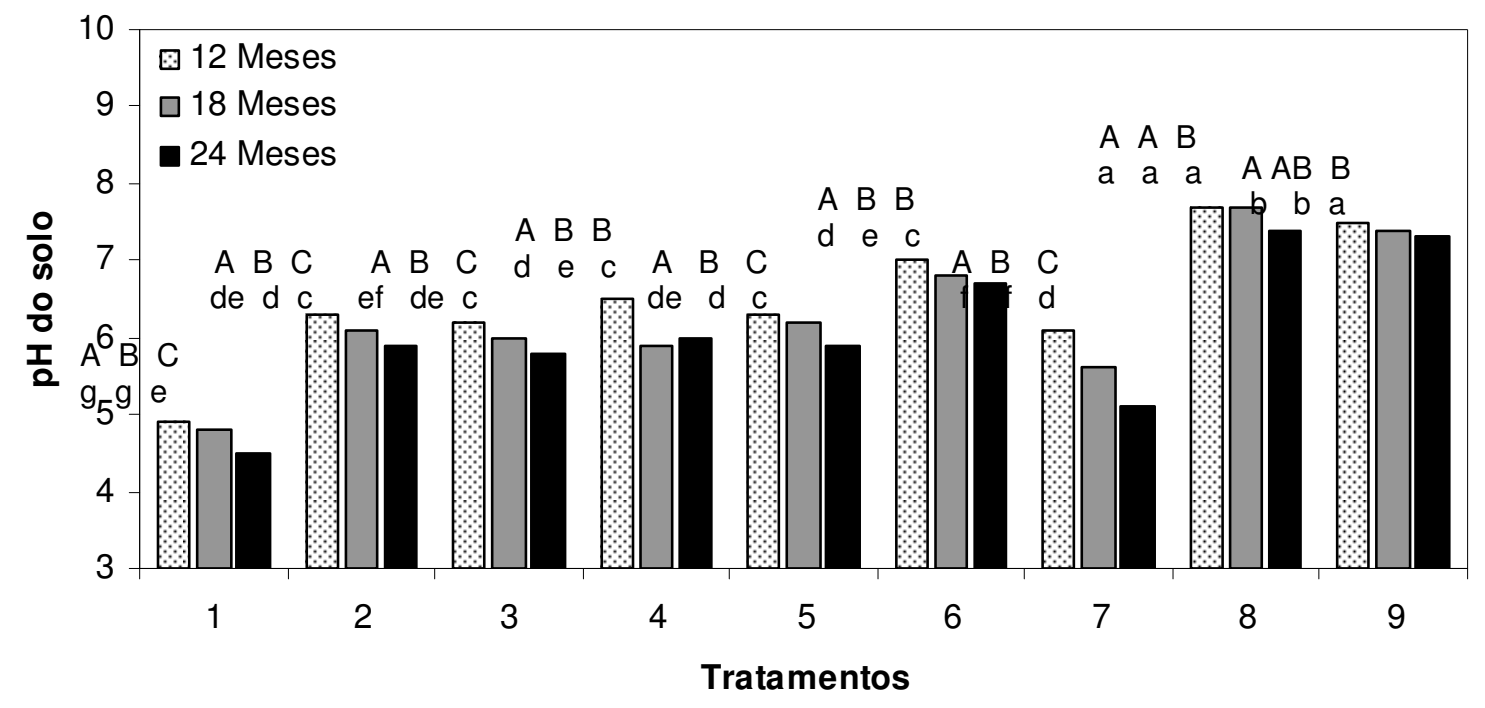

Figura 1. Valor de pH do solo em três épocas de amostragem afetados pela aplicação de escória de siderurgia e calcário. Médias acompanhadas de mesma letra, minúscula entre tratamentos na mesma época e maiúscula entre épocas no mesmo tratamento, não diferem significativamente entre si pelo teste de Tukey (p<0,05). Tratamentos: 1) Controle; 2) $\mathrm{CaCO}_{3}+\mathrm{MgCO}_{3}$; 3) $\mathrm{CaCO}_{3}+\mathrm{MgCO}_{3}+\mathrm{NPK}$; 4) Escória nível 1; 5) Escória nível 1+ NPK; 6) Escória nível 2 + NPK; 7) Escória nível $1+$ N; 8) Escória nível $5+\mathrm{T} 3$; 9) Escória nível $10+\mathrm{T} 3$

Houve diferença significativa entre os tratamentos 5 e 6 quanto à correção do $\mathrm{pH}$ do solo nas três épocas de amostragem (Figura 1). Esta diferença deve-se principalmente à maior dosagem de escória aplicada no tratamento 6. Observa-se ainda que o material com partículas entre $0,149 \mathrm{~mm}$ e 0,5 $\mathrm{mm}$ deste tratamento reagiu no solo, porém esta fração de partículas não assegurou maior poder residual ao material, pois houve diminuição do $\mathrm{pH}$ do solo com o passar do tempo de aplicação, em ambos os tratamentos.

A escória, assim como o carbonato, aumentou a CTC efetiva do solo (Tabela 4). A elevação do $\mathrm{pH}$ do solo aumenta a CTC devido a ação das cargas dependentes de $\mathrm{pH}$ do solo (MEURER et al., 2004). Nota-se, no presente trabalho, que os maiores valores de CTC efetiva ocorreram nos tratamentos com os maiores valores de $\mathrm{pH}$. Isto é devido à maior quantidade de cargas negativas nos solos com $\mathrm{pH}$ mais elevado. Em geral, os horizontes superficiais dos solos apresentam um ponto de carga zero (PCZ) baixo, devido à presença de matéria orgânica, o que indica o predomínio de cargas negativas numa ampla faixa de $\mathrm{pH}$, favorecendo a adsorção de cátions (MEURER et al., 2004). Entre as épocas, praticamente não se observou diferenças na CTC efetiva do solo. Apenas nos tratamentos 7, 8 e 9, ocorreram reduções significativas, acompanhando os resultados encontrados nestes tratamentos para os valores de $\mathrm{pH}$ entre épocas (Figura 1). Resultados semelhantes foram obtidos por Peregrina et al. (2008) que observaram que após dois anos da aplicação da escória de aciaria não foram observadas diferenças entre a CTC efetiva.

Para a CTC a pH 7,0, as diferenças entre os tratamentos foram menores e em vários casos de tratamentos com corretivos não diferiram significativamente do tratamento controle. Comparativamente à CTC efetiva, estes resultados eram previstos, considerando-se o valor de $\mathrm{pH}(7,0)$ em que é realizada esta determinação, tendendo-se a expressar o potencial de cargas do solo independentemente dos tratamentos. Como os tratamentos alteraram o pH do solo, consequentemente as cargas são afetadas e quando se avalia a CTC a pH 7,0, o efeito dos tratamentos sobre o pH é minimizado (WANG et al., 2011). Possivelmente pelo mesmo motivo, também não foram observadas diferenças para esta determinação para cada tratamento entre as três épocas. 
A saturação por bases foi afetada pela adição do carbonato e da escória (Tabela 4). Os maiores valores ocorreram nos tratamentos que apresentaram maior $\mathrm{pH}$ e, ao mesmo tempo, maiores teores de $\mathrm{Ca}$ e $\mathrm{Mg}$. Este é um indicador indireto da condição de acidez, apresentando alta correlação com $\mathrm{pH}$ do solo. Resultados semelhantes foram encontrados por Prado; Fernandes (2001), em trabalho onde avaliaram a resposta da cana-deaçúcar à adição de escória como corretivo da acidez.

Em duas épocas de amostragem, aos 12 e 24 meses após o início do experimento, foram avaliados os teores de manganês, cobre, zinco, cádmio, cromo e níquel no solo pelo método EPA 3050B (USEPA, 1996a). Embora não constituam propriamente os teores totais, pois o método extrai as quantidades de nutrientes no solo ambientalmente disponíveis, estes valores podem ser considerados como representativos da quase totalidade destes elementos no solo. O uso de extrator fitodisponível ou extrator das quantidaes disponíveis, como EDTA ou $\mathrm{HCl}, 1,0 \mathrm{M}$, apenas identificam o potencial disponível para as plantas e não indicam o potencial disponível em termos ambientais (LIU et al., 2014).

De modo geral, houve aumento nos teores de $\mathrm{Mn}, \mathrm{Cu}$ e $\mathrm{Cr}$ no solo nos tratamentos que receberam escória em ambas as épocas, principalmente nos níveis 1 e 2 (Tratamentos 4, 5, 6 e 7), os quais apresentam granulometria mais fina (Tabela 5). Para o $\mathrm{Ni}$ aos 12 meses, o aumento foi significativo apenas no nível 2 (tratamento 6). Neste tratamento, o aporte destes metais foi o dobro em relação aos tratamentos que receberam o nível 1. Quanto aos teores de $\mathrm{Zn}$ e Cd, não foram observadas diferenças significativas, comparando-se os tratamentos com e sem escória, dentro de cada época.

À exceção do $\mathrm{Cd}$, observou-se o aumento significativo dos teores dos demais elementos dos 12 aos 24 meses, principalmente para os tratamentos com maiores doses de escória (8 e 9) (Tabela 5). Este fato, aliado aos maiores teores no tratamento 6, com granulometria mais fina, já aos 12 meses, é um indicativo de que o método utilizado pode não determinar as quantidades totais destes metais no solo, incluindo as partículas do resíduo. Outra possibilidade seria a separação das partículas mais grosseiras de escória quando da amostragem do solo, subestimando assim sua contribuição na primeira época pelo menor tempo de reação.

Dentre os metais analisados, além do Ferro $(\mathrm{Fe}), \mathrm{Mn}$ e $\mathrm{Cr}$ tiveram as maiores concentrações na escória de aciaria utilizada no experimento (Tabela 1), o que gerou um grande aporte destes metais mediante a aplicação dos tratamentos com este resíduo. Assim, os teores destes elementos no solo foram aumentados pela adição de escória. Para a primeira época de avaliação (12 meses), os maiores aumentos foram nos tratamentos que receberam escória fina e no tratamento 6, com granulometria intermediária, porém com o dobro da dose (nível 2). Na avaliação entre épocas, observa-se aumento significativo no teor total de $\mathrm{Mn}$ e $\mathrm{Cr}$ no solo apenas nos tratamentos com partículas mais grosseiras (8 e 9). Houve também uma maior liberação destes metais por parte do tratamento 8 , ao final dos 24 meses. Isto demonstra que as partículas de tamanho entre $0,5 \mathrm{~mm}$ e $2,0 \mathrm{~mm}$ reagiram mais rapidamente no solo do que o material mais grosseiro (2,0 a 15,0 mm) do tratamento 9 .

Com relação ao Níquel, outro elemento presente na escória em quantidade relativamente alta (Tabela 2), não houve aumento significativo dos teores totais aos 12 meses, com exceção ao tratamento 6. Mas avaliando-se ao final de 24 meses, observa-se aumento significativo do teor no solo, principalmente quando da aplicação das maiores doses.

Os resultados demonstram que a escória básica de aciaria tem potencial de contaminação do solo por metais pesados potencialmente tóxicos. Outros estudos mostram que escorias em geral quando adicionadas ao ambiente sem monitoramento ou controle, podem contaminar o meio ambiente (GUARCIA-GUINEA et al., 2010). Porém, é necessário o aprofundamento dos estudos da liberação de metais presentes no resíduo, pois, como observado neste trabalho, os resultados obtidos podem ser mascarados por imperfeições do método de avaliação, que podem estar relacionados a capacidade extrativa do método e das ligações químicas que os metais apresentam no resíduo, reduzindo a eficiência de extração (NAVARRO et al. 2011; McBRIDE et al., 2011). Também há que se analisar qual a contribuição do tempo de incubação no solo na solubilização dos metais presentes nos resíduos de diferentes tamanhos de partículas. 
Tabela 4. Valores de CTC efetica, CTC a pH 7,0 e saturação por bases (V) do solo em três épocas de amostragem (médias de quatro repetições).

\begin{tabular}{|c|c|c|c|c|c|c|c|c|c|}
\hline \multirow{3}{*}{ TRATAMENTO } & \multicolumn{3}{|c|}{ CTC $_{\text {efetiva }}$} & \multicolumn{3}{|c|}{$\mathrm{CTC}_{\mathrm{pH} 7,0}$} & \multicolumn{3}{|c|}{$\mathrm{V}$} \\
\hline & \multicolumn{9}{|c|}{ Época de amostragem (meses) } \\
\hline & 12 & 18 & 24 & 12 & 18 & 24 & 12 & 18 & 24 \\
\hline & \multicolumn{6}{|c|}{ 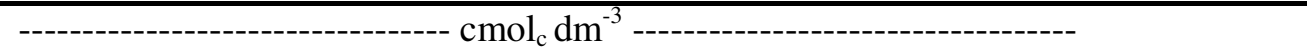 } & \multicolumn{3}{|c|}{--------------- \% ------------- } \\
\hline 1 - Controle & $3,4 \mathrm{~d} A$ & 3,4 e $\mathrm{A}$ & $3,2 \mathrm{~d} \mathrm{~A}$ & $9,3 \mathrm{bcd}$ & $10,0 \mathrm{ab}$ & $9,7 \mathrm{a}$ & 27 e $\mathrm{A}$ & $25 \mathrm{f} \mathrm{B}$ & 23 e $\mathrm{C}$ \\
\hline $2-\mathrm{CaCO}_{3}+\mathrm{MgCO}_{3}$ & 5,7 с C & $6,0 \mathrm{c} \mathrm{AB}$ & $5,8 \mathrm{c} \mathrm{BC}$ & $8,1 \mathrm{de}$ & 8,3 de & $8,1 \mathrm{c}$ & $71 \mathrm{~d} \mathrm{~A}$ & 72 с A & $72 \mathrm{c} \mathrm{A}$ \\
\hline $3-\mathrm{CaCO}_{3}+\mathrm{MgCO}_{3}+\mathrm{NPK}$ & 5,6 c A & $5,8 \mathrm{c} \mathrm{A}$ & 5,7 c A & $7,9 \mathrm{e}$ & $8,3 \mathrm{de}$ & $8,4 \mathrm{bc}$ & $71 \mathrm{~d} \mathrm{~A}$ & $70 \mathrm{c} \mathrm{A}$ & $68 \mathrm{c} \mathrm{B}$ \\
\hline 4 - Escória nível 1 & 6,4 c A & 6,3 c A & $6,0 \mathrm{c} \mathrm{B}$ & 8,5 cde & 8,7 cde & $8,3 \mathrm{c}$ & 76 c A & 72 c B & 72 c B \\
\hline 5 - Escória nível $1+$ NPK & 6,2 c A & 6,2 c A & $6,0 \subset \mathrm{A}$ & 8,4 cde & $8,5 \mathrm{de}$ & $8,4 \mathrm{bc}$ & $74 \mathrm{~cd} \mathrm{~A}$ & 73 c A & $71 \mathrm{c} \mathrm{B}$ \\
\hline 6 - Escória nível $2+$ NPK & $8,1 \mathrm{~b} \mathrm{~A}$ & $7,5 \mathrm{~b} \mathrm{~B}$ & $7,9 \mathrm{~b} \mathrm{~A}$ & $9,6 \mathrm{abc}$ & $9,0 \mathrm{bcd}$ & $9,5 \mathrm{ab}$ & 84 b A & $83 \mathrm{~b} \mathrm{~A}$ & $84 \mathrm{~b} \mathrm{~A}$ \\
\hline 7 - Escória nível 1 + N & $5,6 \mathrm{c} \mathrm{A}$ & $4,7 \mathrm{~d} \mathrm{C}$ & 5,2 c B & $7,9 \mathrm{e}$ & $7,7 \mathrm{e}$ & $8,3 \mathrm{bc}$ & $71 \mathrm{~d} \mathrm{~A}$ & 61 e B & $63 \mathrm{~d} \mathrm{~B}$ \\
\hline 8 - Escória nível 5 + T3 & 9,7 a $\mathrm{A}$ & 9,5 a $\mathrm{AB}$ & 9,3 a B & $10,6 \mathrm{a}$ & $10,5 \mathrm{a}$ & $10,3 \mathrm{a}$ & 91 a A & 91 a A & 91 a $\mathrm{A}$ \\
\hline 9 - Escória nível $10+\mathrm{T} 3$ & 9,0 a $\mathrm{A}$ & 8,7 a $A B$ & $8,6 \mathrm{ab} \mathrm{B}$ & $10,1 \mathrm{ab}$ & $9,8 \mathrm{abc}$ & $9,7 \mathrm{a}$ & 90 a $\mathrm{A}$ & 89 a A & 89 a A \\
\hline
\end{tabular}

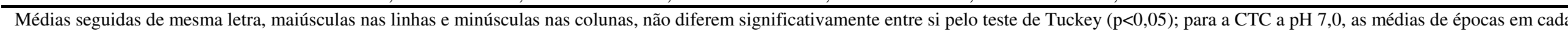
tratamento não diferem entre si. 
Tabela 5. Concentrações de metais no solo relacionadas aos tratamentos avaliadas em duas épocas (médias de quatro repetições).

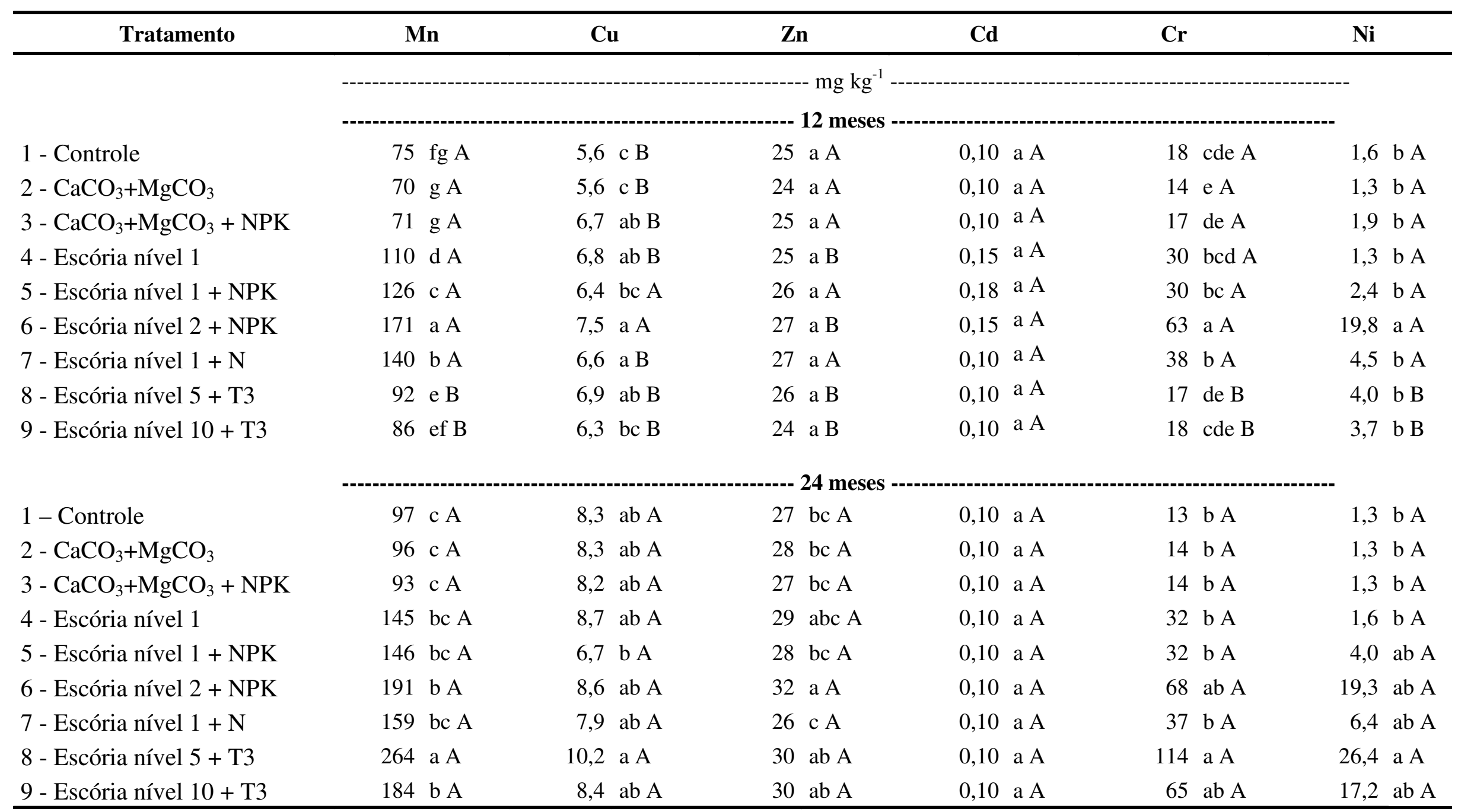

Nas colunas, médias seguidas de mesma letra, minúsculas entre tratamentos na mesma época e maiúsculas entre épocas no mesmo tratamento, não diferem significativamente entre si pelo teste de Tukey $(p<0,05)$. 
Alta dispersão de argilas em horizontes transicionais, coincidindo com a maior expressão do caráter coeso, foi verificada em Latossolos e Argissolos originados de sedimentos provenientes da Formação Barreiras (MOUREAU et al., 2006; CORRÊA et al., 2008a; LIMA NETO et al., 2009). Ainda, a iluviação das argilas dispersas provocando o entupimento dos poros, tem sido apontada como um importante fator na gênese dos horizontes coesos (FONSECA, 1986; CORRÊA et al., 2008b; LIMA NETO et al., 2010). Cunha et al. (2003) caracterizando a matéria orgânica de Latossolos Amarelos coesos com e sem horizonte $\mathrm{A}$ húmico dos Tabuleiros Costeiros do Recôncavo Baiano, verificaram uma maior proporção de frações orgânicas pouco polimerizadas e altamente móveis no solo com A húmico. Estes autores atribuíram a esta fração orgânica a grande translocação de argilas para horizontes subsuperficiais neste solo, e a consequente desorganização estrutural do mesmo.

\section{CONCLUSÕES}

A escória básica de aciaria finamente moída teve efeito similar ao carbonato na correção do $\mathrm{pH}$ do solo, no aumento da CTC efetiva e da saturação de bases.

Partículas com diâmetro até $15 \mathrm{~mm}$ também foram efetivas para aumentar os valores desses atributos.

As maiores doses de escória aumentaram os teores de $\mathrm{Mn}, \mathrm{Cr}$ e $\mathrm{Ni}$ no solo em relação ao tratamento controle.

A escória básica apresenta potencial para a utilização na agricultura como corretivo de solo e fonte de nutrientes, porém é necessário o monitoramento sobre as concentrações de metais pesados e seus efeitos no solo e ambiente, evitando o desequilíbrio ambiental. Além disso, altas doses de escória de aciaria devem ser evitadas para não ocorrer desequilíbrio químico do solo.

\begin{abstract}
As the majority of industrial processes, siderurgy activities generate large amounts of residues. The siderurgic slags, byproducts of steel production have chemical characteristics that indicate their possible use in agriculture, as liming materials and sources of some plant nutrients. However, they may enclose undesired components, like heavy metals, which may result in situations of environmental hazard. In order to evaluate the effect on soil chemical properties and heavy metals accumulation in the soil by the application of steel basic slag, an experiment was carried out in soil columns (Typic Paleudult; $10 \mathrm{dm}^{3} /$ column soil volume), under natural rainfall conditions, in a completely randomized design with four replications. Soil treatments based on the liming rate do achieve pH 6,5 by the SMP method were: T1) Control; T2) $\mathrm{CaCO}_{3}+\mathrm{MgCO}_{3}$ (neutralizing value - PN 100\%); T3) Treat. 2 + NPK fertilizers; T4) Slag - 1 x rate (particles < 0,149 mm e PN 60\%); T5) Treat. 4 + NPK; T6) Slag - 2 x rate (1/3 particles < 0,149 mm e 2/3 particles 0,149 $-0,5 \mathrm{~mm})+\mathrm{NPK}$; T7) Treat. 4 + N fertilizer; T8) Slag $-5 \mathrm{x}$ rate (particles 0,5 - 2,0 mm) + Treat. 3; T9) Slag - 10 x rate (particles 2,0-15,0 mm) + Treat. 3. At equivalent rates both slag and carbonate increased similarly the values of $\mathrm{pH}$, effective CEC and bases saturation in the soil. These attributes were higher for the treatments with slag at higher rates and larger particle size denoting the reactivity of the coarse factions. Among the analyzed metals, only $\mathrm{Mn}, \mathrm{Cr}$ and $\mathrm{Ni}$ at higher slag rates $(2,5$ and $10 \mathrm{x})$ increased in the soil to values significantly higher than the control and carbonate treatments. The results show that the used scoria is efficient as liming material and that it is not expected to provide excessive heavy metals accumulation when applied at rates to rise $\mathrm{pH}$ value up to 6,5 in soils with similar characteristics to that of this study. In summary, the slag exhibited a high potential for $\mathrm{Ph}$ corrections in soil.
\end{abstract}

KEYWORDS: Soil acidity. Industrial residue. Nutrients.

\title{
REFERÊNCIAS
}

ARRIVABENE, L. F.; PINTO JUNIOR, L. A. B.; OLIVEIRA, J. R.; TENÓRIO, J. A. S.; ESPINOSA, D. C. R. Viabilidade técnica da fabricação de cimento com mistura de escória de aciaria LD e resíduo de granito.

Revista Escola de Minas, Ouro Preto, v. 65, p. 241-246, 2012.

BARBER, S. Liming materials and practices. In: PEARSON, R. W.; ADAMS, F. (Eds) Soil acidity and liming. Madison, ASA, 1967. p. 125-160. (Agronomy Series, 12)

BISSANI, C. A.; MEURER, E. J; BOHNEN, H. Solos ácidos e solos afetados por sais. In: MEURER, E.J. (ed) Fundamentos de química do solo. 2 ed. Porto Alegre, Genesis, 2004. p. 181-205. 
CAMARGO, A. P. Influência da granulometria de três materiais corretivos na neutralização da acidez do solo. 1972, 59f. Dissertação (Mestrado em Solos e Nutrição Mineral de Plantas) - Escola Superior de Agricultura "Luiz de Queiroz", Universidade de São Paulo, Piracicaba, 1972.

FORTES, J. L. O. Eficiência de duas escórias de siderurgia, do Estado do Maranhão, na correção da acidez do solo. 1993, 66f. Dissertação (Mestrado em Solos e Nutrição de Plantas) - Universidade Federal de Viçosa, Viçosa, 1993.

GARCIA-GUINEA, J.; CORRECHER, V.; RECIO-VAZQUEZ, L.; CRESPO-FEO, E.; GONZALEZMARTIN, R.; TORMO, L. Influence of accumulation of heaps of steel slag on the environment: determination of heavy metals content in the soils. Anais da Academia Brasileira de Ciências, Rio de Janeiro, v. 82, p. 267$277,2010$.

GUIMARÃES, J. E. P. Escórias na fertilidade do solo. Instituto Geográfico e Geológico de São Paulo. Brasil, 1967. 42p.

LIU, J.; SAMPLE, D. J.; OWEN, J. S.; LI, J.; EVANYLO, G. Assessment of selected bioretention blends for nutrient retention using mesocosm experiments. Journal of Environmental Quality, v. 43, p. 1754-1763, 2014. http://dx.doi.org/10.2134/jeq2014.01.0017

LUZ, P. C.; TEDESCO, M. J.; VILELA, A. C. F. Avaliação de corretivos da acidez de três solos do Rio Grande do Sul. In: SEMINÁRIO NACIONAL DE RESíDUOS SÓLIDOS, 4. GRAMADO-RS. Anais. Gramado-RS, 2002.

McBRIDE, M. B.; MATHUR, R. R.; BAKER, L. L. Chemical extractability of lead in field-contaminated soils: implications for estimating total lead. Communications in Soil Sciemce and Plant Analysis, Athens, v. 42, p. 1581-1593, 2011. http://dx.doi.org/10.1080/00103624.2011.581729

MEURER, E. J.; RHEINHEIMER, D.; BISSANI, C. A. Fenômenos de sorção em solos. In: MEURER, E.J. (ed) Fundamentos de química do solo. 2 ed. Porto Alegre, Genesis, 2004. p. 131-179.

NAVARRO, E. M. G.; TAGLE, M. E. V.; MARIN, M. T. L.; ALFONSO, M. S. P. Comparison of USEPA 3050B and ISO 14869-1:2001 digestion methods for sediment analysis by using FAAS and ICP-OES quantification techniques. Química Nova, São Paulo, v. 34, p. 1443-1449, 2011. http://dx.doi.org/10.1590/S010040422011000800025

NEGIN, O.; ELOIFI, B.; MENCH, M.; BES, C.; GASTE, H.; MOTELICA-HEINO, M.; LE COUSTUMER, P. Effect of basic slag addition on soil properties, growth and leaf mineral composition of beans in a $\mathrm{Cu}-$ contaminated soil. Soil and Sediment Contamination: An International Journal, Amherst, v. 19, p. 174197, 2010. http://dx.doi.org/10.1080/15320380903548508

PEREGRINA, F.; MARISCAL, I.; ORDONEZ, R; GONZALEZ, P.; TEREFE, T.; ESPEJI, R. Agronomic implications of converter basic slag as a magnesium source on acid soils. Soil Science Society of American Journal, Madison, v. 72, p. 402-411. 2008.

PINTO JUNIOR, L. A. B.; VIEIRA, E. A.; TENÓRIO, J. A. S.; PEIXOTO, R. A. F.; OLIVEIRA, J. R. Caracterização da mistura de resíduos de granito e escória de aciaria LD. Revista Escola de Minas, Ouro Preto, v. 64, p. 169-174, 2011.

PRADO, R. M.; KORNDORFER, G. H. Efeitos da escória de siderurgia sobre a cultura do milho (Zea mays L.) cultivado em um Latossolo Vermelho amarelo distrófico. Científica, Jaboticabal, v. 31, p. 9-17, 2003.

PRADO, R. M.; FERNANDES, F. M.; NATALE, W. Uso agrícola da escória de siderurgia no Brasil: estudos na cultura da cana-de-açúcar. Jaboticabal: FUNEP, 2001. 67p. 
ROSSA JR., J.; PORTELLA, K. F. Adição de escória de alto forno em argamassas colantes tipo AC-I. Cerâmica, São Paulo, v. 58, 542-548, 2012.

SBCS/NRS. Recomendações de adubação e calagem para os Estados do Rio Grande do Sul e Santa Catarina. 3 ed. Passo Fundo: Embrapa - CNPT/Núcleo Regional Sul, 1995. 224p.

STRECK, E. V.; KAMPF, N.; DALMOLIN, R. S. D., KLAMT, E.; NASCIMENTO,P. C.; SCHENEIDER, P. Solos do Rio Grande do Sul. $2^{\text {a }}$ Ed. Porto Alegre: Editora da UFRGS, 2008. 222p.

TEDESCO, M. J.; GIANELLO, C; BISSANI, C. A; BOHNEN, H; VOLKWEISS, S. J. Análise de solo, plantas e outros materiais. 2.ed Porto Alegre: Depto de Solos da UFRGS. 1995. 174p. (Boletim Técnico de Solos $\left.n^{\circ} 5\right)$.

USEPA. Method 3050B. Acid digestion of sediments, sludges, and soils. In: Test methods for evaluating solid waste. Report SW - 846. USEPA. Revision 2. 1996a. p. 3050-1 - 3050-12. CD-ROM.

USEPA. Method 3052. Microwave assisted acid digestion of siliceous and organically based matrices. In: Test methods for evaluating solid waste. Report SW - 846. USEPA. Revision 0. 1996b. p. 3052-1 - 3052-20. CDROM.

VALADARES, J. M. A. S.: BATAGLIA, O. C.: FURLANI, P. R. Estudos de materiais calcários usados como corretivos do solo no Estado de São Paulo. Determinação de Mo, Cu, Zn e Fe. Bragantia, Campinas, v. 33, p. 147-152, 1974. http://dx.doi.org/10.1590/S0006-87051974000100015

WANG, G.; WANG, Y.; GAO, Z. Use of steel slag as a granular material: volume expansion prediction and usability criteria. Journal of Hazardous Material, New York, v. 184, p. 555-560, 2011.

http://dx.doi.org/10.1016/j.jhazmat.2010.08.071 